\title{
Economic impact of a genetic test for cisplatin-induced ototoxicity
}

\author{
F Dionne, C Mitton, R Rassekh, \\ B Brooks, C Ross, M Hayden \\ and B Carleton
}

The Pharmacogenomics Journal (2012) 12, 359; doi:10.1038/tpj.2011.21

Correction to: The Pharmacogenomics Journal (2012) 12, 205-213; doi:10.1038/ tpj.2011.15; published online 19 April 2011

The above article was published with incomplete affiliations. The authors apologize for this error. The authors, with the correct affiliations, are listed below.

F Dionne ${ }^{1}$, C Mitton $^{1,2}$, R Rassekh ${ }^{3}$, B Brooks ${ }^{4,5}$, C Ross $^{6,7}$, M Hayden $^{6,7}$ and B Carleton ${ }^{6,8,9}$

${ }^{1}$ Centre for Clinical Epidemiology and Evaluation, VCHRI, Vancouver, Canada; ${ }^{2}$ School of Population and Public Health, University of British Columbia, Vancouver, Canada; ${ }^{3}$ Department of Pediatrics, Division of Pediatric Hematology/Oncology/BMT, British Columbia Children's Hospital, Vancouver, Canada; ${ }^{4}$ Audiology and Speech Pathology Department, British Columbia's Children's Hospital, Vancouver, Canada; ${ }^{5}$ School of Audiology and Speech Sciences, University of British Columbia, Vancouver, Canada; ${ }^{6} \mathrm{Child}$ and Family Research Institute, Children's and Women's Health Research Centre of B.C., Vancouver, Canada; ${ }^{7}$ Department of Medical Genetics, University of British Columbia, Centre for Molecular Medicine and Therapeutics, Vancouver, Canada; ${ }^{8}$ Faculty of Pharmaceutical Sciences, University of British Columbia, Vancouver, Canada and ${ }^{9}$ Department of Paediatrics, Pharmaceutical Outcomes Programme, University of British Columbia, Vancouver, Canada 